\title{
OVERUSE OF PRESCRIPTION AND OTC NON-STEROIDAL ANTI-INFLAMMATORY DRUGS IN PATIENTS WITH RHEUMATOID ARTHRITIS AND OSTEOARTHRITIS
}

\author{
L. CAVAGNA ${ }^{1}$, R. CAPORALI ${ }^{1}$, G. TRIFIRÒ' ${ }^{2}$, V. ARCORACI $^{2}$, S. ROSSI $^{1}$ \\ and C. MONTECUCCO ${ }^{1}$
}

\begin{abstract}
${ }^{1}$ Division of Rheumatology, University and IRCCS Foundation Policlinico S. Matteo, Pavia, Italy;
${ }^{2}$ Department of Clinical and Experimental Medicine and Pharmacology, University of Messina, Italy
\end{abstract}

Received September 13, 2012 - Accepted February 7, 2013

Non-steroidal anti-inflammatory drugs (NSAIDs) have been demonstrated to have significant cardiovascular and gastrointestinal toxicity; high dose of intake and concomitant use of multiple compounds or corticosteroids are factors that increase the risk of NSAID toxicity. In this paper we described our experience on NSAIDs misuse (both prescribing and OTC formulations), particularly relevant in the setting of rheumatoid arthritis (39.5\% of patients) and osteoarthritis (47\% of patients). We also evaluated causes underlying NSAIDs misuse (e.g. not satisfactory pain control, other painful conditions, etc).

Non-steroidal-anti-inflammatory drugs (NSAIDs) are commonly used for the treatment of chronic diseases such as osteoarthritis (OA) and rheumatoid arthritis (RA). These drugs have been demonstrated to have significant cardiovascular $(1,2)$ and gastrointestinal toxicity (3), in particular with high dose intake or concomitant use of multiple compounds (4) or corticosteroids (5). NSAIDs are commercially available either as prescription-drug or over-thecounter (OTC) medications. OTC formulations contain lower dosages of the active principle as compared to the corresponding prescription drug, however, this does necessarily correlate with safer patient outcome (6). From January 2012 to September 2012, a convenience sample of consecutive RA and OA patients being followed-up in our outpatient-clinic and for whom we had previously prescribed NSAID were evaluated for NSAID patterns of consumption.
Per clinic protocol, all patients are informed of the safety risks and precautions related to NSAID use. Five hundred forty-six RA and 508 OA were assessed. In all cases the NSAID prescribed in our clinic was taken correctly, according to our indications. In the 2 months preceding the visit, 216 RA patients (39.5\%) concurrently took additional NSAIDs (over-users) and $147(27 \%)$ were simultaneously on chronic corticosteroid treatment. Nimesulide $(\mathrm{N}=90 ; 16 \%)$, acetyl-salicylic-acid $(\mathrm{N}=57 ; 10 \%)$ and OTC ibuprofen or diclofenac ( $\mathrm{N}=105 ; 19 \%)$ were the most frequently associated NSAIDs. Incomplete pain control $(\mathrm{N}=105$; $19 \%)$, headache $(\mathrm{N}=111 ; 20 \%)$, fever $(\mathrm{N}=51 ; 9 \%)$ and dental pain $(\mathrm{N}=24 ; 4 \%)$ were the predominant reasons for additional NSAID treatment as prescribed by other physician or self-administration. Neither gender $(\mathrm{p}=0.0834)$ nor mean age $(\mathrm{p}=0.2564)$ were different between NSAID over-user and non-over-

Key words: non-steroidal anti-inflammatory drugs, over-the-counter, overuse, rheumatoid arthritis, osteoarthritis

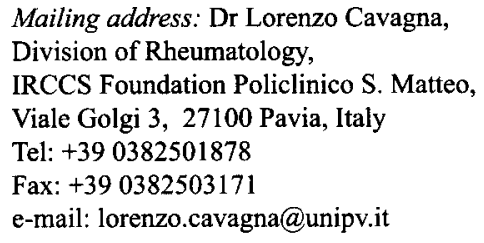

0394-6320 (2013)

Copyright $\mathcal{O}$ by BIOLIFE, s.a.s. This publication and/or article is for individual use only and may not be further reproduced without written permission from the copyright holder Unauthorized reproduction may result in financial and other penalties DISCLOSURE: ALL AUTHORS REPORT NO CONFLICTS OF INTEREST RELEVANT TO THIS ARTICLE. 
Table I. Characteristics of patients evaluated.

\begin{tabular}{|c|c|c|}
\hline & Rheumatoid arthritis & Osteoarthritis \\
\hline Patients' number & 546 & 508 \\
\hline NSAIDs non over-user & $330(60.5)$ & $268(53)$ \\
\hline mean age (years) \pm SD non over-user & $66 \pm 11.2 \S$ & $68.2 \pm 7 £$ \\
\hline Females/male non over-user & $250 / 80^{\wedge}$ & $155 / 113^{\circ}$ \\
\hline NSAIDs over-user (\%) & $216(39.5)$ & $240(47)$ \\
\hline mean age (years) \pm SD over-user & $65 \pm 8 \&$ & $67 \pm 11 £$ \\
\hline Females/males over-user & $149 / 67^{\wedge}$ & $150 / 90^{\circ}$ \\
\hline $\begin{array}{l}\text { NSAIDs over-user with concomitant } \\
\text { corticosteroids (\%) }\end{array}$ & $147(27)$ & $0(0)$ \\
\hline \multicolumn{3}{|l|}{ Reasons for NSAIDs over-use } \\
\hline unsatisfactor pain disease control & $105(19)$ & $104(20)$ \\
\hline headhache $(\%)$ & $111(20)$ & $56(11)$ \\
\hline fever & $51(9)$ & $56(11)$ \\
\hline dental pain $(\%)$ & $24(4.5)$ & $48(9)$ \\
\hline other reasons* $(\%)$ & $0(0)$ & $10(2)$ \\
\hline more than 1 reason $(\%)$ & $57(10)$ & $48(9)$ \\
\hline \multicolumn{3}{|l|}{ Associated NSAIDs } \\
\hline nimesulide (\%) & $90(16)$ & $72(14)$ \\
\hline diclofenac $(\%)$ & $12(2)$ & $8(2)$ \\
\hline piroxicam (\%) & $18(3)$ & $4(1)$ \\
\hline COXIBs (\%) & $6(1)$ & $12(3)$ \\
\hline acetyl-salycilic acid (\%) & $57(10)$ & $108(21)$ \\
\hline other prescribing NSAIDs (\%) & $6(1)$ & $0(0)$ \\
\hline total OTC $(\%)$ & $105(19)$ & $80(16)$ \\
\hline OTC ibuprofen (\%) & $81(15)$ & $60(12)$ \\
\hline OTC diclofenac $(\%)$ & $24(4)$ & $20(4)$ \\
\hline
\end{tabular}

* other reasons: post-traumatic pain, menstrual pain, fatigue. ${ }^{\wedge} p=0.0834,{ }^{\circ} p=0.3184, \S p=0.2564, £ p=0.1367$

user RA patients. In the same period, 240 OA patients $(47 \%)$ took additional NSAIDs as prescribed by other physicians or self-administration. Nimesulide $(\mathrm{N}=72 ; 14 \%)$, acetyl-salycilic-acid $(\mathrm{N}=108 ; 21 \%)$ and OTC ibuprofen or diclofenac (80 patients; 16\%) were the mainly associated NSAIDs. Incomplete pain control (104 patients; 20\%), headache (56 patients; $11 \%$ ), fever (56 patients; $11 \%$ ) and dental pain (48 patients; $9 \%$ ) were the main reasons for additional NSAID use. Sex $(p=0.3184)$ and mean age $(\mathrm{p}=0.1367)$, were not different between NSAID over-user and non-over-user OA patients. Table I characterized differences between $\mathrm{RA}$ and $\mathrm{OA}$ patients. Overall analysis reveals: a) NSAID overuse is common in both RA and OA; b) it involves both prescription and OTC drugs; and c) very importantly, conditions other than disease-related pain may lead to additional NSAID consumption as the result of physician prescription or self-medications. These findings indicate that physicians do not always take into account the pharmaceutical background of patients and patients are not completely aware of risks associated with NSAID use, despite having been previously informed, or perhaps are not familiar with other medications in the family of NSAIDs. Based on these patterns of misuse revealed by this study and others (7), clinicians must enhance their own habits of medication reconciliation in regard to multiple NSAIDs and concomitant glucocorticoid use as well as their approach to patient education in order to 
emphasize safe NSAID use and hopefully reduce the risk of NSAID-related side-effects.

\section{REFERENCES}

1. Atzeni F, Turiel M, Caporali R, Cavagna L, Tomasoni L, Sitia S, Sarzi-Puttini P. The effect of pharmacological therapy on the cardiovascular system of patients with systemic rheumatic diseases. Autoimmun Rev 2010; 9:835-9.

2. Gislason GH, Rasmussen JN, Abildstrom SZ, et al. Increased mortality and cardiovascular morbidity associated with use of nonsteroidal anti-inflammatory drugs in chronic heart failure. Arch Intern Med 2009; 169:141-9.

3. Gupta M, Eisen GM. NSAIDs and the gastrointestinal tract. Curr Gastroenterol Rep 2009; 11:345-53.

4. Bueno H, Bardají A, Patrignani P, Martín-Merino E,
García-Rodríguez LA. Spanish Case-Control Study to Assess NSAID-Associated ACS Risk Investigators. Use of non-steroidal antiinflammatory drugs and type-specific risk of acute coronary syndrome. Am J Cardiol 2010; 105:1102-6.

5. Nielsen G, Sorensen H, Mellemkjoer L, Blot W, McLaughlin J, Tage-Jensen U, Olsen JH. Risk of hospitalisation resulting from upper gastrointestinal bleeding among patients taking corticosteroids: a register-based cohort study. Am J Med 2001; 111:541-5.

6. Lavonas EJ, Fries JF, Furst DE, et al. Comparative risks of non-prescription analgesics: a structured topic review and research priorities. Expert Opin Drug Saf 2012; 1:33-44.

7. Alacqua $M$, Trifirò $G$, Cavagna $L$, et al. Prescribing pattern of drugs in the treatment of osteoarthritis in Italian general practice: the effect of rofecoxib withdrawal. Arthritis Rheum 2008; 59:568-74. 